Methods Medical records of cervical cancer patients who received operation in our institution from January 2007 to December 2018 were retrospectively reviewed. Cases were divided into 2 period groups (group 1, 2007-2013, and group 2, 2014-2018), based on the date of operation. Between the two groups, clinical outcomes, including clinicopathologic variables, surgical methods, operative details, adjuvant treatments, 3-year recurrence rates and disease-free survivals (DFS) were compared.

Results A total of 331 cervical cancer patients were included in the study analysis, 224 patients in group 1 and 107 in group 2. Overall, minimally invasive surgery (MIS) was more frequently performed in group $2(56.3 \%$ vs. $69.2 \%$, $\mathrm{p}=0.025$ ), especially in earlier stages (stage IA, 69.0\% vs. $100.0 \%$; stage IB1, $52.9 \%$ vs. $67.3 \%$ ). However, the mean tumor size of stage IB cervical cancer cases treated by MIS was significantly smaller in group 2 (23.6 vs. $17.7 \mathrm{~mm}$, $\mathrm{p}=0.019$ ). In addition, adjuvant treatment was less frequently performed in group 2, especially in stage IB1 $(52.9 \%$ vs $32.7 \%, \mathrm{p}=0.015)$. There was a trend of decreased 3-year recurrence rates $(8.5 \%$ in group 1 vs. $4.7 \%$ in group 2, $\mathrm{p}=0.211$ ).

Conclusions Institutional quality control monitoring positively affected clinical outcomes of cervical cancer patients.

\section{EPV068/\#398 RANDOMIZED CONTROLLED TRIAL OF THE EFFICACY OF ADJUVANT CHEMOTHERAPY IN PATIENTS WITH RESIDUAL LESIONS AFTER CONCURRENT CHEMORADIATION THERAPY FOR LOCALLY ADVANCED CERVICAL CANCER (CQG0G0102)}

M He*, Z Lin, H Wang, Q Zhou, D Zou. Chongqing University Cancer Hospital, The Gynecologic Oncology Center, Chongqing, China

\subsection{6/ijgc-2021-IGCS.136}

Objectives The aim of this trial is to compare response rate and survivals of locally advanced stage cervical cancer patients with residual lesions who had Concurrent Chemoradiation therapy (CCRT) alone to those who had adjuvant chemotherapy after CCRT.

Methods The CQGOG0102 study is a single-center, randomized controlled trial. The patients who have residual lesions after CCRT are randomized to arm A by observation or arm B by adjuvant chemotherapy with paclitaxel plus cisplatin every 3 weeks for 3 cycles.

Results In our center, a retrospective study found that residual lesion after CCRT was one of the most important prognostic factors in patients with LACC. PFS and OS was decreased when the size of the residual lesion was over $10 \mathrm{~mm}$. A

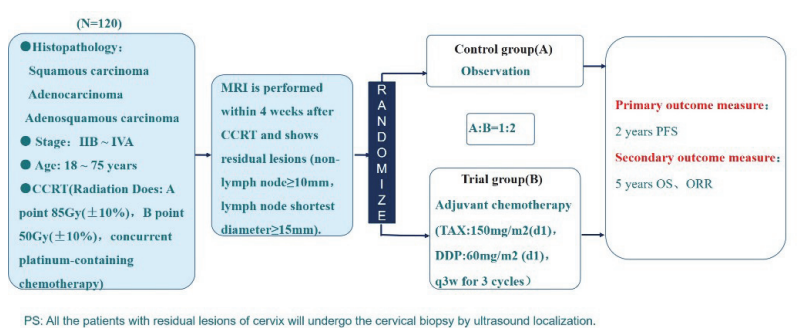

Abstract EPV068/\#398 Figure 1 Study design
Abstract EPV068/\#398 Table 1 Brief inclusion and exclusion criteria

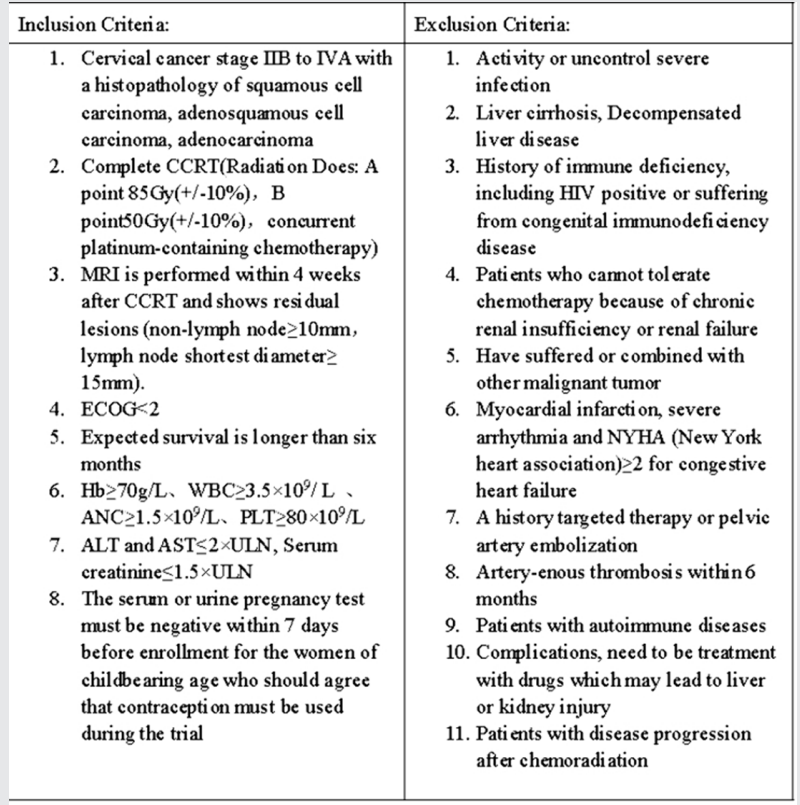

further study showed that patients with residual lesion after CCRT treated with ACT had a significantly longer PFS compared to patients without ACT $(22.4 \mathrm{~m}$ vs. $12 \mathrm{~m}, \mathrm{p}<0.05)$ So, we designed the randomized controlled trial, CQGOG0102, to evaluate the efficacy of ACT in LACC with residual lesions after CCRT. At present, 30 patients have been enrolled. Pathological evidence of cervical residual lesion was identified in $23.3 \%(7 / 30)$. This trial is currently open and enrolling patients.

Conclusions ACT may improve the prognosis of LACC who has the residual lesion after CCRT. We will report the primary, midterm and final results about this study in the future. Clinical trial information: NCT04409860

\section{EPV069/\#40 SYSTEMATIC COMPARISON OF INTERNATIONAL TREATMENT GUIDELINES FOR LOCALLY ADVANCED CERVICAL CANCER}

${ }^{1}$ E Pujade-Lauraine* ${ }^{2}$ A Leary, ${ }^{3}$ J Takyar, ${ }^{4}$ A Nunes, ${ }^{4}$ JD Hernandez Chagui, ${ }^{4} K$ Rabon-Stith, ${ }^{5}$ B Monk. 'Arcagy-Gineco, Medical Oncology, Paris, France; ${ }^{2}$ Gustave Roussy Cancer Center, INSERM U981, and Groupe d'Investigateurs Nationaux pour I'Etude des Cancers Ovariens (GINECO), Gynecological Unit, Villejuif, France; ${ }^{3}$ Parexel International, Evidence and Heor, Chandigarh, India; ${ }^{4}$ AstraZeneca, Global Medical Affairs, Gaithersburg, USA; ${ }^{5}$ Arizona Oncology (US Oncology Network), Gynecologic Oncology, Obstetrics and Gynecology, Phoenix, USA

\subsection{6/ijgc-2021-IGCS.137}

Objectives Globally, cervical cancer is a leading cause of death. Lack of international consensus on standard-of-care (SoC) treatment for locally advanced cervical cancer (LACC) (Stages IB2-IVA) may contribute to inconsistent treatment. We compared LACC treatment recommendations from international guidelines.

Methods Literature databases (1999-2020), national authority websites, and bibliographies were searched for English-language cervical cancer guidelines, with no restriction on 